\title{
Functional Approach for Solving Reduced Order of Index-Four Hessenberg Differential-Algebraic Control System
}

\author{
Ghazwa F. Abd (1) \\ Department of Mathematics, College of Science, Mustansiriyah University, Baghdad, Iraq \\ Correspondence should be addressed to Ghazwa F. Abd; k.abd@uomustansiriyah.edu.iq
}

Received 13 November 2021; Accepted 6 January 2022; Published 31 January 2022

Academic Editor: Fazlollah Soleymani

Copyright (@) 2022 Ghazwa F. Abd. This is an open access article distributed under the Creative Commons Attribution License, which permits unrestricted use, distribution, and reproduction in any medium, provided the original work is properly cited.

\begin{abstract}
This research investigates differential-algebraic equations with higher index (index four). Specifically, a functional analytic approach is proposed to find the solution of (index four) Hessenberg differential-algebraic equations (DAEs). The approximate solution of the proposed functional approach for a suitable separable Hilbert space is obtained with the help of the direct optimisation technique and Ritz basis functions method. Illustrations have been ranked from an $8 \times 8$ test system of index-four Hessenberg linear DAEs to a $4 \times 4$ DAE of rotating masses as well as an $8 \times 8$ differential-algebraic generator model, where it reformulated index-four linear Hessenberg DAEs. Their approximate solutions were obtained using the present approach with comparisons. The numerical results demonstrate the simplicity of the proposed approach and express suitable accuracy and efficiency.
\end{abstract}

\section{Introduction}

Many life applications can be modelled as differential-algebraic systems, including optimal control problems, constrained robotic systems, and constrained electrical networks [1-3]. In the literature, the index property, of which several definitions exist, is usually utilised to characterise the differential-algebraic system $[4,5]$. To determine an equivalent ordinary differential equation, the minimum number of times that the algebraic constraint of DAEs must be differentiated to time is represented with the frequently used differentiation index [5]. The higher index DAEs present a greater challenge in being solved than those in the lower index system because it makes applications more cumbersome [6]. In general DAEs, with a special structure, Hessenberg DAEs are an exceedingly important class implemented in various engineering and scientific applications $[7,8]$.

The authors in $[2,3,9]$ presented numerical methods to solve index two and index three. These methods utilize the index reduction method to reduce the higher DAE index to be lower one [10-12].

To solve higher index (index four) Hessenberg linear DAE systems, this article develops an analytical approximation method to be efficiently and easily implemented. It is based on the functional analytical theory, which will help to determine a functional with critical points that serve as solutions for the fourth index Hessenberg DAEs and vice versa. To present the solution as a linear combination of the basic elements of the given setting space (i.e., separable Banach space), this work introduces a parameterisation approach inserted into the obtained variational formulation. Subsequently, to determine the solution of the variational formulation for unknown parameters, the method uses a direct method of calculus of variation. Finally, solving the linear algebraic system obtains the unknown parameters.

\section{Fourth-Order Hessenberg DAEs Problem Formulation}

Index-four linear Hessenberg DAEs with control input are considered as follows:

$$
\begin{array}{r}
\dot{x}_{1}+A_{11} x_{1}+A_{12} x_{2}+A_{13} x_{3}+A_{14} x_{4}=B_{1} u+f_{1}, \\
\dot{x}_{2}+A_{21} x_{1}+A_{22} x_{2}+A_{23} x_{3}=B_{2} u+f_{2}, \\
\dot{x}_{3}+A_{32} x_{2}+A_{33} x_{3}=B_{3} u+f_{3},
\end{array}
$$




$$
A_{43} x_{3}=B_{4} u+f_{4} \text {, }
$$

where

(1) $A_{43} A_{32} A_{21} A_{14} \triangleq c$ is a square invertible matrix, and $\left(x_{1}, x_{2}, x_{3}, x_{4}\right)^{T}$ denotes independent state vectors

(2) $A_{i j} ; i, j=1, . ., 4$, are constant matrices of appropriate dimension, and $A_{i j}$ is not required to be invertible or a square matrix

(3) $B_{j}, j=1, . ., 4$ are suitable constant vectors, and $u$ is the given single control input with $u \in C^{3}[T ; R] \triangleq$ $\nu_{u}$ as the class of admissible control. $T=\left[t_{0}, t_{f}\right]$, $t_{0}<t_{f} ;$ and $f_{j} ; j=1, . ., 4$ denotes differentiable functions, $f_{j} \in C^{3}[T ; R]$

Using the implicit function theorem, which can be observed in $[13,14]$, (1d) can be differentiated with respect to $t$ :

$$
A_{43} \dot{x}_{3}=B_{4} \dot{u}+\dot{f}_{4} .
$$

From equation (1c), the following is obtained:

$$
-B_{4} \dot{u}-\dot{f}_{4}+A_{43}\left[B_{3} u+f_{3}-A_{32} x_{2}-A_{33} x_{3}\right]=0 .
$$

Now, in equation (3), a new constraint is conjugated with equations (1a)-(1d) (i.e., differential-algebraic system). The differentiability of equation (3) continues until the state $x_{4}$ is estimated to have a class of differential equations with states $x_{1}, x_{2}, x_{3}$ defined on a manifold of the resulting algebraic constraints.

Furthermore, $x_{4}$ (state variable) does not exist in equation (3); accordingly, equation (1c) can be differentiated (with respect to $t$ ) again.

$$
\begin{aligned}
& -B_{4} \ddot{u}-\ddot{f}_{4}+A_{43}\left[B_{3} \dot{u}+\dot{f}_{3}-A_{32} \dot{x}_{2}-A_{33} \dot{x}_{3}\right]=0 \\
& -B_{4} \ddot{u}+A_{43} B_{3} \dot{u}-\left(A_{43} A_{32} B_{2}+A_{43} A_{33} B_{3}\right) u-\ddot{f}_{4}+A_{43} \dot{f}_{3}-A_{43} A_{32} f_{2}-A_{43} A_{33} f_{3}+A_{43} A_{32} A_{21} x_{1} \\
& \quad+\left(A_{43} A_{32} A_{22}+A_{43} A_{33} A_{32}\right) x_{2}+\left(A_{43} A_{32} A_{23}+A_{43} A_{33} A_{32}\right) x_{3}=0 .
\end{aligned}
$$

Hence, another algebraic constraint (inserted into the differential-algebraic system (1a)-(1d)) is represented by equation (5). To determine the state variable $x_{4}$ and until the index-four condition $\left(A_{43} A_{32} A_{21} A_{14}\right)$ is satisfied, the derivation is continued:

$$
\begin{aligned}
& -B_{4} \stackrel{\dot{\mathfrak{u}}}{ }+A_{43} B_{3} \ddot{u}-\left(A_{43} A_{32} B_{2}+A_{43} A_{33} B_{3}\right) \dot{u}+\left(A_{43} A_{32} A_{21} B_{1}+A_{43} A_{32} A_{22} B_{2}+\right. \\
& \left.\cdot A_{43} A_{33} A_{32} B_{2}+A_{43} A_{32} A_{23} B_{3}+A_{43} A_{33} A_{32} B_{3}\right) u-f_{4}+A_{43} \ddot{f}{ }_{3}-A_{43} A_{32} \dot{f}_{2}+ \\
& \cdot A_{43} A_{32} A_{21} f_{1}\left(A_{43} A_{32} A_{22}+A_{43} A_{33} A_{32}\right) f_{2}+\left(A_{43} A_{32} A_{23}+A_{43} A_{33} A_{32}\right) f_{3}- \\
& \cdot\left(A_{43} A_{32} A_{21} A_{11}+A_{43} A_{32} A_{22} A_{21}+A_{43} A_{33} A_{32} A_{21}\right) x_{1}- \\
& \cdot\left(A_{43} A_{32} A_{21} A_{12}+A_{43} A_{32} A_{22} A_{22}+A_{43} A_{33} A_{32} A_{22}+A_{43} A_{32} A_{23} A_{32}+A_{43} A_{32} A_{23} A_{32}\right) x_{2}- \\
& \cdot\left(A_{43} A_{32} A_{21} A_{13}+A_{43} A_{32} A_{22} A_{23}+A_{43} A_{33} A_{32} A_{23}+A_{43} A_{32} A_{23} A_{33}+A_{43} A_{33} A_{32} A_{33}\right) x_{3}- \\
& \cdot\left(A_{43} A_{32} A_{21} A_{14}\right) x_{4}=0 .
\end{aligned}
$$

Then, since $A_{43} A_{32} A_{21} A_{14}$ is invertible,

$$
x_{4}=c^{-1}\left(\widetilde{u}+\widetilde{f}+\widetilde{A}_{1} x_{1}+\widetilde{A}_{2} x_{2}+\widetilde{A}_{3} x_{3}\right)=L\left(x_{1}\right)+L\left(x_{2}\right)+L\left(x_{3}\right)+L(\widetilde{u}, \widetilde{f}),
$$

where $c=A_{43} A_{32} A_{21} A_{14}, L\left(x_{1}\right)=c^{-1} \widetilde{A}_{1} \quad x_{1}, L\left(x_{2}\right)=c^{-1} \widetilde{A}_{2}$ $x_{2}, L\left(x_{3}\right)=c^{-1} \widetilde{A}_{3} x_{3}, L(\widetilde{u}, \widetilde{f})=c^{-1}(\widetilde{u}+\widetilde{f})$,

$$
\begin{aligned}
\widetilde{u}= & -B_{4} \stackrel{\dot{\mathfrak{u}}}{u}+A_{43} B_{3} \ddot{u}-\left(A_{43} A_{32} B_{2}+A_{43} A_{33} B_{3}\right) \dot{u} \\
& +\left(A_{43} A_{32} A_{21} B_{1}+A_{43} A_{32} A_{22} B_{2}+A_{43} A_{33} A_{32} B_{2}+A_{43} A_{32} A_{23} B_{3}+A_{43} A_{33} A_{32} B_{3}\right) u
\end{aligned}
$$




$$
\begin{aligned}
& \tilde{f}=-\stackrel{\mathfrak{t}}{f}_{4}+A_{43} \ddot{f}_{3}-A_{43} A_{32} \dot{f}_{2}+A_{43} A_{32} A_{21} f_{1}+\left(A_{43} A_{32} A_{22}+A_{43} A_{33} A_{32}\right) f_{2}+\left(A_{43} A_{32} A_{23}+A_{43} A_{33} A_{32}\right) f_{3}, \\
& \tilde{A}_{1}=-\left(A_{43} A_{32} A_{21} A_{11}+A_{43} A_{32} A_{22} A_{21}+A_{43} A_{33} A_{32} A_{21}\right), \\
& \widetilde{A}_{2}=-\left(A_{43} A_{32} A_{21} A_{12}+A_{43} A_{32} A_{22} A_{22}+A_{43} A_{33} A_{32} A_{22}+A_{43} A_{32} A_{23} A_{32}+A_{43} A_{32} A_{23} A_{32}\right), \\
& \widetilde{A}_{3}=-\left(A_{43} A_{32} A_{21} A_{13}+A_{43} A_{32} A_{22} A_{23}+A_{43} A_{33} A_{32} A_{23}+A_{43} A_{32} A_{23} A_{33}+A_{43} A_{33} A_{32} A_{33}\right) .
\end{aligned}
$$

Since system (1) is the index-four Hessenberg DAEs system, the initial condition must be selected in such a way as to satisfy all algebraic constraints in equations (1d), (3), and (5). From the additional two hidden constraint equations (1c)-(1d) and from equation (7), over the class of $C^{3}[T ; R]$, the following form is the reduced type index-four Hessenberg DAEs (1) with control $u, \dot{u}, \ldots, u$.

$$
\begin{gathered}
\dot{x}_{1}+\left(A_{11}+A_{14} c^{-1} \widetilde{A}_{1}\right) x_{1}+\left(A_{12}+A_{14} c^{-1} \widetilde{A}_{2}\right) x_{2} \\
+\left(A_{13}+A_{14} c^{-1} \widetilde{A}_{3}\right) x_{3}=B_{1} u+f_{1}-A_{14} L(\widetilde{u}, \widetilde{f}), \\
\dot{x}_{2}+A_{21} x_{1}+\cdots+A_{23} x_{3}=B_{2} u+f_{2}, \\
\dot{x}_{3}+A_{32} x_{2}+A_{33} x_{3}=B_{3} u+f_{3},
\end{gathered}
$$

with algebraic constraint one : $A_{43} x_{3}=B_{4} u+f_{4}$,

algebraic constraint two : $A_{43} A_{32} x_{2}+A_{43} A_{33} x_{3}$

$$
=-B_{4} \dot{u}+A_{43} B_{3} u-\dot{f}_{4}+A_{43} f_{3} \text {, }
$$

algebraic constraint three : $A_{43} A_{32} A_{21} x_{1}+\widetilde{A}_{2} x_{2}+\widetilde{A}_{3} x_{3}$

$$
\begin{aligned}
= & B_{4} \ddot{u}-A_{43} B_{3} \dot{u} \\
& +\left(A_{43} A_{32} B_{2}+A_{43} A_{33} B_{3}\right) u+\ddot{f}_{4} \\
& -A_{43} \dot{f}_{3}+A_{43} A_{32} f_{2}+A_{43} A_{33} f_{3} .
\end{aligned}
$$

Set $\mathfrak{\Im}=\left\{\left(x_{1}(),. x_{2}(),. x_{3}().\right) \in C^{3}\left(T ; R^{3}\right) \mid\right.$ where $(12)-(14)$ are satisfied. Ultimately, the following represents the class of consistent initial condition:

$$
J^{0}=\left\{\left(x_{1}\left(t_{0}\right), x_{2}\left(t_{0}\right), x_{3}\left(t_{0}\right)\right) \in R^{3} \mid J \text { is satisfied at } t=t_{0}\right\} \text {. }
$$

\section{Fourth Index Hessenberg DAE Solution via Variational Formulation Technique}

The present approach is step by step represented as follows:

Step 1: define the operator $L: D(L) \subset H \longrightarrow R(L)$ C $H$, with $\overline{D(L)}=H$, where $H$ is an appropriate Hilbert space (e.g., $H \triangleq L_{2}(T) ; T=\left[t_{0}, t_{f}\right], t_{0}<t_{f}$ ) by $L x=F(t ; \tilde{u}, \widetilde{f})$. The operator $L$ refers to a linear differential and algebraic operator can be written as follows:

$$
L x=\left[\begin{array}{lll}
I & 0 & 0 \\
0 & I & 0 \\
0 & 0 & I \\
0 & 0 & 0 \\
0 & 0 & 0 \\
0 & 0 & 0
\end{array}\right]\left[\begin{array}{l}
\frac{d x_{1}}{d t} \\
\frac{d x_{2}}{d t} \\
\frac{d x_{3}}{d t}
\end{array}\right]+\left[\begin{array}{ccc}
A_{1} & \mathscr{A}_{2} & \mathscr{A}_{3} \\
A_{21} & A_{22} & A_{23} \\
0 & A_{21} & A_{21} \\
0 & 0 & A_{21} \\
0 & A_{4} & \mathscr{A}_{5} \\
\mathscr{A}_{6} & A_{21} & A_{21}
\end{array}\right]\left[\begin{array}{c}
x_{1} \\
x_{2} \\
x_{3}
\end{array}\right] .
$$

where $\quad \mathscr{A}_{1}=A_{11}+A_{14} c^{-1} \widetilde{A}_{1}, \quad \mathscr{A}_{2}=A_{12}+A_{14} c^{-1}$ $\widetilde{A}_{2}, \mathscr{A}_{3}=A_{13}+A_{14} c^{-1} \widetilde{A}_{3}, \quad \mathscr{A}_{4}=A_{43} A_{32}, \mathscr{A}_{5}=A_{43}$ $A_{33}, \mathscr{A}_{6}=A_{43} A_{32} A_{21}$, and $F(t ; \widetilde{u}, \widetilde{f}) \triangleq F(t ; u, \dot{u}, \ddot{u}, u$, $\left.f_{j}, \dot{f}_{j}, \dot{f}_{j}, f_{j}^{(3)}\right)$, for all $u \in C^{3}[T ; R] \triangleq \Delta_{u}$

By using the variational formulation of obtained functional corresponding to (13) on $\mathrm{D}(L)=\{x()=$. $\left(x_{1}(.), x_{2}(.), x_{3}(.)\right)^{T} \in \quad L_{2}\left[T ; R^{3}\right] \mid x(t) \in \mathfrak{J}, x\left(t_{0}\right)$ $\in \mathfrak{F}^{0}$ and $x(t)$ satisfy (13)\}.

In almost everywhere in $H$, the DAEs are satisfied by the linearly invariant (reduced type) index Hessenberg DAEs system. Furthermore, almost everywhere over the setting class $C^{1}\left[T ; R^{3}\right]$, the solution should be a continuous function that satisfies equations (12)-(14))

Step 2: in equation (16), the operator $L$ is not symmetric to the usual bilinear form $(x, \tilde{x})=\int x^{T}$ $(t) . \tilde{x}(t) d t, \quad x=\left(x_{1}(t), x_{2}(t), x_{3}(t)\right), \tilde{x}=\left(\widetilde{x}_{1}(t), \tilde{x}_{2}\right.$ $(t), \tilde{x}_{3}(t)$ because of the existence of the operator $d . / d t$. To satisfy the nondegenerate condition on the range and the domain of operator $L$, the bilinear form needs to be chosen (i.e., for every $x \in D$ $(L),(x, \tilde{x})=0$, then $\tilde{x}=0$, and for every $\tilde{x} \in D$ $(L),(x, \tilde{x})=0 \quad$ (then $x=0)$ ). Therefore, a usual variational formulation exists only if the usual bilinear (inner product) or the linear operator is redefined form using $\langle x, \widetilde{x}\rangle \triangleq(x, L \widetilde{x}), x, \widetilde{x} \in D(L)$, where $D(L) \subset H_{L} \subset H$ with $\|x\|_{L}=\sqrt{(x, L \tilde{x})}$, and $\left(H_{L}, d(x, \tilde{x})\right)$ is the complete Hilbert space (where $L$ refers to the asymmetric linear operator and separable Hilbert space is denoted by $H_{L}$ ). The reader is recommended to $[10,11]$ for more details. $J(x)$ is defined if $L$ (on $\overline{D(L)}$ ) is positive definite; otherwise, it is needed to determine $J_{\widetilde{J}^{0}}(x)$ (the critical points). Over the class of the consistent initial conditions and 
$u \in C^{3}[T ; R]$, the augmented functional of $J_{\mathfrak{\mho}^{0}}(x)$ can be defined as follows:

$$
\begin{aligned}
J_{\widetilde{J}^{0}}(x)= & \frac{1}{2}\left(L_{1}^{T} x(t), L_{1} x(t)\right)-\left(F_{1}^{T}, L_{1} x(t)\right)+\cdots+\frac{1}{2}\left(L_{6}^{T} x(t), L_{6} x(t)\right)-\left(F_{6}^{T}, L_{6} x(t)\right)+\frac{1}{2}\left(L_{4}^{T} x\left(t_{0}\right), L_{4} x\left(t_{0}\right)\right) \\
& -\left(F_{4}^{T}\left(t_{0}\right), L_{4} x\left(t_{0}\right)\right)+\frac{1}{2}\left(L_{5}^{T} x\left(t_{0}\right), L_{5} x\left(t_{0}\right)\right)-\left(F_{5}^{T}\left(t_{0}\right), L_{5} x\left(t_{0}\right)\right)+\frac{1}{2}\left(L_{6}^{T} x\left(t_{0}\right), L_{6} x\left(t_{0}\right)\right)-\left(F_{6}^{T}\left(t_{0}\right), L_{6} x\left(t_{0}\right)\right) .
\end{aligned}
$$

where $L_{1} x(t)=\dot{x}_{1}+\mathscr{A}_{1} x_{1}+\mathscr{A}_{2} x_{2}+\mathscr{A}_{3} x_{3}, L_{2} x(t)$ $=\dot{x}_{2}+A_{21} x_{1}+\cdots+A_{23} x_{3}, L_{3} x(t)=\dot{x}_{3}+A_{32} x_{2}+A_{33}$ $x_{3}, L_{4} x(t)=A_{43} x_{3}, L_{5} x(t)=\mathscr{A}_{4} x_{2}+\mathscr{A}_{5} x_{3}, L_{6} x(t)=$ $\mathscr{A}_{6} x_{1}+\mathscr{A}_{2} x_{2}+\mathscr{A}_{3} x_{3}$, and $F_{1}=B_{1} u+f_{1}-A_{14} L$ $(\tilde{u}, \tilde{f}), F_{2}=B_{2} u+f_{2}, F_{3}=B_{3} u+f_{3}, F_{4}=B_{4} u+f_{4}$, $F_{5}=-B_{4} \dot{u}+A_{43} B_{3} u-\dot{f}_{4}+A_{43} f_{3}$, and $F_{6}=B_{4} \ddot{u}-$ $A_{43} B_{3} \dot{u}+\left(\mathscr{A}_{4} B_{2}+\mathscr{A}_{5} B_{3}\right) u+f_{4}-A_{43} \dot{f}_{3}+\mathscr{A}_{4} f_{2}+$ $\mathscr{A}_{5} f_{3}$.

Thus, the critical points of $J_{\mathfrak{I}^{0}}(x)$ is guaranteed by the functional as the solution of equations (12)-(14) and vice versa when $(x, \tilde{x})$ is chosen in the form of nondegenerate bilinear.

Step 3: the critical points of equations (12)-(14) are the solutions of equation (12). Furthermore, the critical points are a solution of equations (12)-(14) by the nondegenerate property on $D(L), R(L)$. A countable linearly independent (bases) system $\left(\varphi_{1}\right.$, $\varphi_{2}, \ldots$ etc.) (complete set of functions; base) can be used to approximate the solution, where $x_{j}(t)=$ $\sum_{\zeta=1}^{n} a_{\zeta} \varphi_{\zeta}, n$ is a positive number, $j=1,2,3$ and $a_{\zeta}$ is determined so that $J\left(x_{1}(t), x_{2}(t), x_{3}(t)\right)$ is extremized.

Step 4: with respect to $a_{\zeta}, \forall \zeta=1, \ldots, n, J$ can be derived. To determine these critical points as a function of $a_{\zeta}$ parameters, $n$ is given a suitable positive number. To obtain the system of a solvable linear algebraic, one can set $d J / d a_{\zeta}=0, \forall \zeta=1, . ., n$. Furthermore, $\sum_{\zeta=1}^{n} \mathrm{M}(\zeta, j) a_{\zeta}=\mathrm{N}_{j}, \forall j=1, \ldots, n$, $M=(\mathrm{M}(\zeta, j))_{\zeta=1, j=1}^{n, 3}$ is a nonsingular matrix, where the coefficients can be determined from $d J / d a_{\zeta}=0$, if $x\left(t_{0}\right) \in \mathfrak{J}^{0}$. Accordingly, $a_{\zeta}=\mathrm{M}^{-1}(\zeta, j) \mathrm{N}_{j}$, and the unique solution is guaranteed. Else, if $x\left(t_{0}\right)$ is chosen as arbitrary, there may be infinitely solutions or no solution.

\section{Illustration Examples}

4.1. Test Example. The following test example is designed with a suitable exact solution in mind to confirm the validity of the aforementioned approach, where

(1) The system is $8 \times 8$ DAEs with control

(2) The system is represented as fourth order Hessenberg linear DAE with control

$$
\begin{aligned}
\dot{x}_{11}+2 x_{11}+3 x_{12}+x_{31}+x_{41} & =f_{11}, \\
\dot{x}_{12}-2 x_{11}-2 x_{21}+x_{22}+x_{31}+x_{41} & =u+f_{12}, \\
\dot{x}_{21}+4 x_{11}+x_{12}-2 x_{21}+x_{31} & =3 u+f_{21}, \\
\dot{x}_{22}+x_{12}-2 x_{21}+x_{32} & =f_{22} \\
\dot{x}_{31}+3 x_{21}-2 x_{22}+x_{31} & =f_{31}, \\
\dot{x}_{32}+3 x_{21}+x_{32} & =-2 u+f_{32}, \\
x_{31} & =-u+f_{42}, \\
x_{32} & =f_{42},
\end{aligned}
$$

with

$$
\begin{aligned}
& f_{11}=t^{4}+t+3, f_{12}=t^{5}+t^{3}-t^{2}-\sin t-4, \\
& f_{21}=5 t+t^{4}-2 t^{2}-3 \sin t-2, f_{22}=t^{5}+t^{3}-2 t^{2}-t+1, \\
& f_{31}=t^{4}+4 t^{3}+3 t^{2}-4 t+4, f_{32}=t^{5}+5 t^{4}+t^{3}+6 t^{2}+2 \sin t+3, \\
& f_{41}=t^{4}-\sin t-1, f_{42}=t^{5}+t^{3}, \\
& A_{11}=\left[\begin{array}{cc}
2 & 3 \\
-2 & 0
\end{array}\right], A_{12}=\left[\begin{array}{cc}
0 & 0 \\
-2 & 1
\end{array}\right], A_{13}=\left[\begin{array}{ll}
1 & 0 \\
0 & 1
\end{array}\right], A_{14}=\left[\begin{array}{ll}
1 & 0 \\
0 & 1
\end{array}\right], A_{21}=\left[\begin{array}{ll}
4 & 1 \\
0 & 1
\end{array}\right],
\end{aligned}
$$




$$
\begin{aligned}
& A_{22}=\left[\begin{array}{ll}
-2 & 0 \\
-2 & 0
\end{array}\right], A_{23}=\left[\begin{array}{ll}
1 & 0 \\
0 & 1
\end{array}\right], A_{32}=\left[\begin{array}{ll}
3 & -2 \\
3 & 0
\end{array}\right], A_{33}=\left[\begin{array}{ll}
1 & 0 \\
0 & 1
\end{array}\right], A_{43}=\left[\begin{array}{ll}
1 & 0 \\
0 & 1
\end{array}\right], \\
& B_{1}=\left[\begin{array}{l}
0 \\
1
\end{array}\right], B_{2}=\left[\begin{array}{l}
3 \\
0
\end{array}\right], B_{3}=\left[\begin{array}{c}
0 \\
-2
\end{array}\right], B_{4}=\left[\begin{array}{c}
-1 \\
0
\end{array}\right], x_{1}=\left[\begin{array}{l}
x_{11} \\
x_{12}
\end{array}\right], x_{2}=\left[\begin{array}{l}
x_{21} \\
x_{22}
\end{array}\right], x_{3}=\left[\begin{array}{l}
x_{31} \\
x_{32}
\end{array}\right], x_{4}=\left[\begin{array}{l}
x_{41} \\
x_{42}
\end{array}\right] .
\end{aligned}
$$

Note that $\left(A_{43} A_{32} A_{21} A_{14}\right)$ is an invertible matrix.

Significantly, in the DAE system, variable $x_{4}$ does not appear. Therefore, the algebraic constraint equations (18)-(23) can be differentiated with respect to $t$. Then, to obtain the following, substitute the differential constraints to equations (24) and (25). where $A_{4}=A_{43} A_{32}, A_{4}=A_{43} A_{33}$.

Next, one can differentiate equation (28) again for the $t$ set: $c \triangleq\left(A_{43} A_{32} A_{21} A_{14}\right)^{-1}=\left[\begin{array}{cc}0.1250 & -0.0417 \\ -0.5 & 0.5\end{array}\right]$.
Thus, equation (27) is considered a new constraint inserted to equations (18)-(25). In the last constraint, variable $x_{4}$ is not exist. Therefore, to obtain the following, equation (27) can be differentiated one more time with respect to $t$.
Step 1: $x_{4}$ is estimated as $x_{4}=L\left(x_{1}, x_{2}, x_{3}\right)+L(u, \dot{u}$, $\left.\ddot{u}, \stackrel{u}{u}, f_{1}, f_{2}, f_{3}, \dot{f}_{2}, \dot{f}_{3}, \ddot{f}_{3}\right)$, where the derivative of the class of the consistent initial conditions can be

$$
\mathfrak{J}^{0}=\left\{\begin{array}{c}
\left(x_{1}\left(t_{0}\right), x_{2}\left(t_{0}\right), x_{3}\left(t_{0}\right)\right) \mid \\
A_{43} x_{3}\left(t_{0}\right)=B_{4} u\left(t_{0}\right)+f_{4}\left(t_{0}\right) \\
-A_{43} A_{32} x_{2}\left(t_{0}\right)-A_{43} A_{33} x_{3}\left(t_{0}\right)=B_{4} \dot{u}\left(t_{0}\right)+\dot{f}_{4}\left(t_{0}\right)-A_{43} B_{3} u\left(t_{0}\right)-A_{43} f_{3}\left(t_{0}\right) \\
\mathscr{A}_{4} A_{21} x_{1}\left(t_{0}\right)+\mathscr{A}_{5} A_{22} x_{2}\left(t_{0}\right)+\mathscr{A}_{4} A_{23} x_{3}\left(t_{0}\right)+\mathscr{A}_{5} A_{32} x_{2}\left(t_{0}\right)+\mathscr{A}_{5} A_{33} x_{3}\left(t_{0}\right) \\
=B_{4} \ddot{u}\left(t_{0}\right)+\ddot{f}_{4}\left(t_{0}\right)-A_{43} B_{3} \dot{u}\left(t_{0}\right)-A_{43} \dot{f}_{3}\left(t_{0}\right)+\mathscr{A}_{4} B_{2} u\left(t_{0}\right)+\mathscr{A}_{4} f_{2}\left(t_{0}\right)+\mathscr{A}_{5} B_{3} u\left(t_{0}\right)+\mathscr{A}_{5} f_{3}\left(t_{0}\right)
\end{array}\right\} .
$$

Step 2: as can be seen in the following, the definition of the variational function with the class of the consistent initial conditions can be written as follows:

$$
\begin{aligned}
J \mathfrak{J}^{0}[x]= & \int_{t_{0}}^{t_{f}} \frac{1}{2}\left[\left[\dot{x}_{1}+\mathscr{A}_{1} x_{1}+\mathscr{A}_{2} x_{2}+\mathscr{A}_{3} x_{3}\right]^{T}\left[\dot{x}_{1}+\mathscr{A}_{1} x_{1}+\mathscr{A}_{2} x_{2}+\mathscr{A}_{3} x_{3}\right]\right. \\
& +\left[\dot{x}_{2}+\mathscr{A}_{21} x_{1}+\mathscr{A}_{22} x_{2}+\mathscr{A}_{23} x_{3}\right]^{T}\left[\dot{x}_{2}+\mathscr{A}_{21} x_{1}+\mathscr{A}_{22} x_{2}+\mathscr{A}_{23} x_{3}\right]+\left[\dot{x}_{3}+\mathscr{A}_{32} x_{2}+\mathscr{A}_{33} x_{3}\right]^{T} \\
& \cdot\left[\dot{x}_{3}+A_{32} x_{2}+A_{33} x_{3}\right]+\left[A_{43} x_{3}\right]^{T}\left[A_{43} x_{3}\right]+\left[\mathscr{A}_{4} x_{2}+\mathscr{A}_{5} x_{3}\right]^{T}\left[\mathscr{A}_{4} x_{2}+\mathscr{A}_{5} x_{3}\right]+ \\
& \cdot\left[A_{6} x_{1}+A_{2} x_{2}+\mathscr{A}_{3} x_{3}\right]^{T}\left[A_{6} x_{1}+A_{2} x_{2}+A_{3} x_{3}\right]+\left[A_{43} x_{3}\left(t_{0}\right)\right]^{T}\left[A_{43} x_{3}\left(t_{0}\right)\right]
\end{aligned}
$$




$$
\begin{aligned}
& +\left[A_{4} x_{2}\left(t_{0}\right)+A_{5} x_{3}\left(t_{0}\right)\right]^{T}\left[A_{4} x_{2}\left(t_{0}\right)+A_{5} x_{3}\left(t_{0}\right)\right]+\left[\mathscr{A}_{6} x_{1}\left(t_{0}\right)+\mathscr{A}_{2} x_{2}\left(t_{0}\right)+\mathscr{A}_{3} x_{3}\left(t_{0}\right)\right]^{T} \\
& \left.\cdot\left[\mathscr{A}_{6} x_{1}\left(t_{0}\right)+\mathscr{A}_{2} x_{2}\left(t_{0}\right)+\mathscr{A}_{3} x_{3}\left(t_{0}\right)\right]\right]-\left[\left[F_{1}\right]^{T}\left[\dot{x}_{1}+\mathscr{A}_{1} x_{1}+\mathscr{A}_{2} x_{2}+\mathscr{A}_{3} x_{3}\right]+\right. \\
& \cdot\left[F_{2}\right]^{T}\left[\dot{x}_{2}+A_{21} x_{1}+A_{22} x_{2}+A_{23} x_{3}\right]+\left[F_{3}\right]^{T}\left[\dot{x}_{3}+A_{32} x_{2}+A_{33} x_{3}\right]+\left[F_{4}\right]^{T}\left[A_{43} x_{3}\right]+ \\
& \cdot\left[F_{5}\right]^{T}\left[\mathscr{A}_{4} x_{2}+\mathscr{A}_{5} x_{3}\right]+\left[F_{6}\right]^{T}\left[\mathscr{A}_{6} x_{1}+\mathscr{A}_{2} x_{2}+\mathscr{A}_{3} x_{3}\right]+\left[F_{4}\left(t_{0}\right)\right]^{T}\left[A_{43} x_{3}\left(t_{0}\right)\right] \\
& \left.+\left[F_{5}\left(t_{0}\right)\right]^{T}\left[\mathscr{A}_{4} x_{2}\left(t_{0}\right)+\mathscr{A}_{5} x_{3}\left(t_{0}\right)\right]+\left[F_{6}\left(t_{0}\right)\right]^{T}\left[\mathscr{A}_{6} x_{1}\left(t_{0}\right)+\mathscr{A}_{2} x_{2}\left(t_{0}\right)+\mathscr{A}_{3} x_{3}\left(t_{0}\right)\right]\right] d t,
\end{aligned}
$$

where $F_{1}=B_{1} u+f_{1}-c A_{43} B_{3} \ddot{u}-c A_{43} \ddot{f}_{3}+c B_{4} \stackrel{\dot{\mathrm{t}}}{u}+$ $c f_{4}+c \mathscr{A}_{4} B_{2} \dot{u}+c \mathscr{A}_{4} \dot{f}_{2}-c \mathscr{A}_{4} A_{21} B_{1} u-c \mathscr{A}_{4} A_{21} f_{1}-$ $c \mathscr{A}_{4} A_{22} B_{2} u-c \mathscr{A}_{4} A_{22} f_{2}-c \mathscr{A}_{4} A_{23} B_{3} u-c \mathscr{A}_{4} A_{23} f_{3}+$ $c \mathscr{A}_{5} B_{3} \dot{u}+c \mathscr{A}_{5} \dot{f}_{3}-c \mathscr{A}_{5} A_{32} B_{2} u-c \mathscr{A}_{5} A_{32} f_{2},-c \mathscr{A}_{5}$ $A_{33} B_{3} u-c A_{5} A_{33} f_{3}, F_{2}=B_{2} u+f_{2}, \quad F_{3}=B_{3} u+f_{3}$, $F_{4}=B_{4} u+f_{.4}, \quad F_{5}=B_{4} \dot{u}+\dot{f}_{4}-A_{43} B_{3} u-A_{43} f_{3}$, $F_{6}=B_{4} \ddot{u}+\ddot{f}_{4}-A_{43} B_{3} \dot{u}-A_{43} \dot{f}_{3}+\quad \mathscr{A}_{4} B_{2} u+\mathscr{A}_{4} f_{2}+$ $\mathscr{A}_{5} B_{3} u+\mathscr{A}_{5} f_{3}$.

Step 3: one can set $x_{11}(t)=\sum_{i=0}^{5} a_{i} \varphi_{i}(t), x_{12}(t)=\sum_{i=0}^{5}$ $b_{i} \varphi_{i}(t)$,

$x_{21}(t)=\sum_{i=0}^{5} c_{i} \varphi_{i}(t), x_{22}(t)=\sum_{i=0}^{5} d_{i} \varphi_{i}(t), \quad x_{31}(t)=$ $\sum_{i=0}^{5} v_{i} \varphi_{i}(t)$,

and $x_{32}(t)=\sum_{i=0}^{5} w_{i} \varphi_{i}(t)$, with $\varphi_{i}(t)=t^{i}$ to obtain $x_{4}(t)=L\left(x_{1}, x_{2}, x_{3}, t\right)+L\left(u, \dot{u}, \ddot{u}, \stackrel{\mathrm{t}}{u}, f_{1}, f_{2}, \quad f_{3}, \dot{f}_{2}, \dot{f}_{3}\right.$, $\left.\ddot{f}_{3}\right)$

Step 4: as mentioned above, the functional $J$ is a function of $\vec{a}, \vec{b}, \vec{c}, \vec{d}, \vec{v}$, and $\vec{w}$. As $J(\vec{a}, \vec{b}, \vec{c}$, $\vec{d}, \vec{v}, \vec{w})$ is extremized, one can set $\partial J / \partial \vec{a}=0$, $\partial J / \partial \vec{b}=0, \partial J / \partial \vec{c}=0, \partial J / \partial \vec{d}=0, \partial J / \partial \vec{v}=0$, and $\partial J / \partial \vec{w}$. It leads to the linear algebraic equation $\mathrm{M}(\vec{a} \vec{b} \vec{c} \vec{d} \vec{v} \vec{w})=\mathrm{N}$, which is solvable directly for $(\vec{a} \vec{b} \vec{c} \vec{d} \vec{v} \vec{w})=\mathrm{M}^{-1} \mathrm{~N}$. By deriving $J$ with respect to $\vec{a}, \vec{b}, \vec{c}, \vec{d}, \vec{v}$, and $\vec{w}, M$ (the matrix coefficient) can be determined. Hence, the approximate solution $\left(x_{1}(t), x_{2}(t), x_{3}(t)\right)$ is obtained. The numerical values of the unknown coefficients $\vec{a}, \vec{b}, \vec{c}, \vec{d}, \vec{v}$, and $\vec{w}$ are listed in Table 1 .

Figure 1 depicts the numerical solutions with equality states compared with the exact solution $x_{1}=\left[\begin{array}{c}t \\ 1-t\end{array}\right]$, $x_{2}=\left[\begin{array}{l}t^{2}+1 \\ 2 t-1\end{array}\right], x_{3}(t)=\left[\begin{array}{c}t^{4}-1 \\ t^{3}+t^{5}\end{array}\right]$, and $x_{4}(t)=\left[\begin{array}{c}2 t \\ t^{2}\end{array}\right]$, for a given $u(t) \triangleq \sin t$.

4.2. Application of the Generator Model. Consider the model of a generator described with the following parameters. On the right axis, the input is the angle $\theta$ connected to mass with inertia $K$ which is rotated about the angle $\theta$ with angular velocity $\nu$. The torque acting on the right and left sides are $T_{1}$ and $T_{2}$, respectively. The mass is then connected to the second axis with a generator. The electrical quantities and the variables described in the second axis are then assumed as in [15]. The rest of the electrical circuit includes one inductor and resistor. The model is illustrated in Figure 2.

The system is now modelled using the equations representing the different parts and their connections.

$$
\left[\begin{array}{llllllll}
1 & 0 & 0 & 0 & 0 & 0 & 0 & 0 \\
0 & 1 & 0 & 0 & 0 & 0 & 0 & 0 \\
0 & 0 & 1 & 0 & 0 & 0 & 0 & 0 \\
0 & 0 & 0 & 1 & 0 & 0 & 0 & 0 \\
0 & 0 & 0 & 0 & 1 & 0 & 0 & 0 \\
0 & 0 & 0 & 0 & 0 & 1 & 0 & 0 \\
0 & 0 & 0 & 0 & 0 & 0 & 0 & 0 \\
0 & 0 & 0 & 0 & 0 & 0 & 0 & 0
\end{array}\right]\left[\begin{array}{c}
\dot{T}_{1} \\
\dot{T}_{2} \\
\dot{\mathscr{V}} \\
\dot{\varnothing} \\
\dot{I} \\
\dot{u}_{1} \\
\dot{u}_{2} \\
\dot{u}_{3}
\end{array}\right]+\left[\begin{array}{cccccccc}
1 & 2 & 0 & 0 & 1 & 0 & 1 & 0 \\
-1 & 0 & 1 & 1 & 0 & 1 & 0 & 1 \\
2 & 1 & 1 & 0 & 1 & 0 & 0 & 0 \\
0 & 1 & 1 & 0 & 0 & 1 & 0 & 0 \\
0 & 0 & 1 & -1 & 1 & 0 & 0 & 0 \\
0 & 0 & 1 & 0 & 0 & 1 & 0 & 0 \\
0 & 0 & 0 & 0 & 1 & 0 & 0 & 0 \\
0 & 0 & 0 & 0 & 0 & 1 & 0 & 0
\end{array}\right]\left[\begin{array}{c}
T_{1} \\
T_{2} \\
\mathscr{V} \\
\varnothing \\
I \\
u_{1} \\
u_{2} \\
u_{3}
\end{array}\right]=\left[\begin{array}{c}
0 \\
1 \\
1 \\
0 \\
0 \\
-1 \\
1 \\
0
\end{array}\right] \theta,
$$

with $\theta(t) \triangleq \sin t \in C^{2}[I, R]$ 
TABLE 1: Unknown coefficients of the representation of $x_{1}(t), x_{2}(t)$, and $x_{3}(t)$.

\begin{tabular}{|c|c|c|c|c|c|c|}
\hline Coef. of $x_{11}$ states & $a_{5}$ & $a_{4}$ & $a_{3}$ & $a_{2}$ & $a_{1}$ & $a_{0}$ \\
\hline Numerical values & $1 \times 10^{-39}$ & $-8 \times 10^{-39}$ & $1 \times 10^{-38}$ & $-6 \times 10^{-39}$ & 1 & 0 \\
\hline Coef. of $x_{12}$ states & $b_{5}$ & $b_{4}$ & $b_{3}$ & $b_{2}$ & $b_{1}$ & $b_{0}$ \\
\hline Numerical values & $1 \times 10^{-38}$ & $-3 \times 10^{-38}$ & $3 \times 10^{-38}$ & $-1 \times 10^{-38}$ & -1 & 1 \\
\hline Coef. of $x_{21}$ states & $c_{5}$ & $c_{4}$ & $c_{3}$ & $c_{2}$ & $c_{1}$ & $c_{0}$ \\
\hline Numerical values & $2 \times 10^{-38}$ & $-7 \times 10^{-38}$ & $6 \times 10^{-38}$ & 1 & $1 \times 10^{-39}$ & 1 \\
\hline Coef. of $x_{22}$ states & $d_{5}$ & $d_{4}$ & $d_{3}$ & $d_{2}$ & $d_{1}$ & $d_{0}$ \\
\hline Numerical values & $1 \times 10^{-40}$ & $-1 \times 10^{-39}$ & $1 \times 10^{-39}$ & $-1 \times 10^{-39}$ & 2 & -1 \\
\hline Coef. of $x_{31}$ states & $v_{5}$ & $v_{4}$ & $v_{3}$ & $v_{2}$ & $v_{1}$ & $v_{0}$ \\
\hline Numerical values & $2 \times 10^{-40}$ & 1 & $-3 \times 10^{-39}$ & $2 \times 10^{-39}$ & $-3 \times 10^{-40}$ & -1 \\
\hline Coef. of $x_{32}$ states & $w_{5}$ & $w_{4}$ & $w_{3}$ & $w_{2}$ & $w_{1}$ & $w_{0}$ \\
\hline Numerical values & 1 & $1 \times 10^{-38}$ & 1 & $9 \times 10^{-39}$ & $-1 \times 10^{-39}$ & 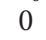 \\
\hline
\end{tabular}

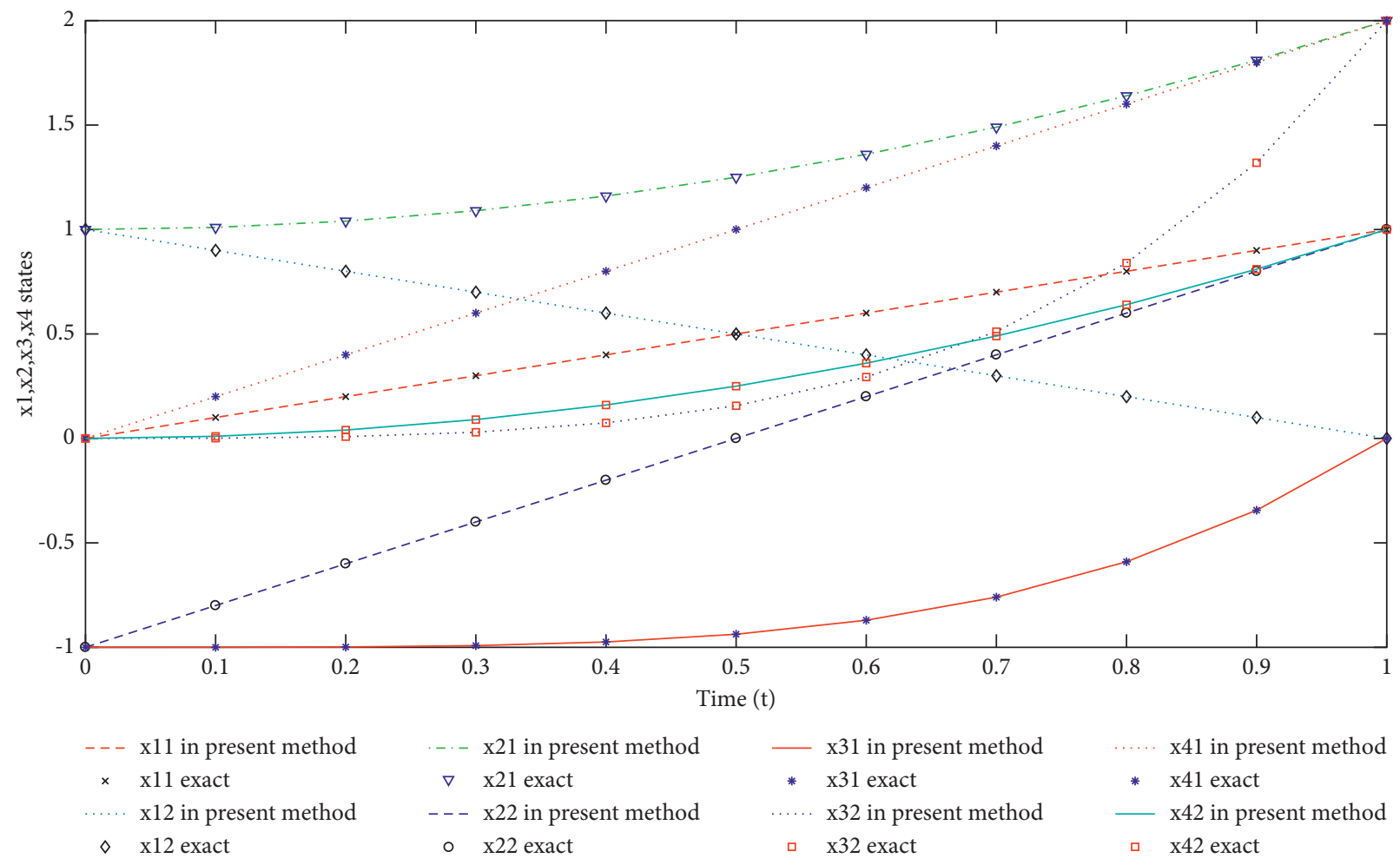

Figure 1: The comparisons between the exact solution and approximate solution of the test example.

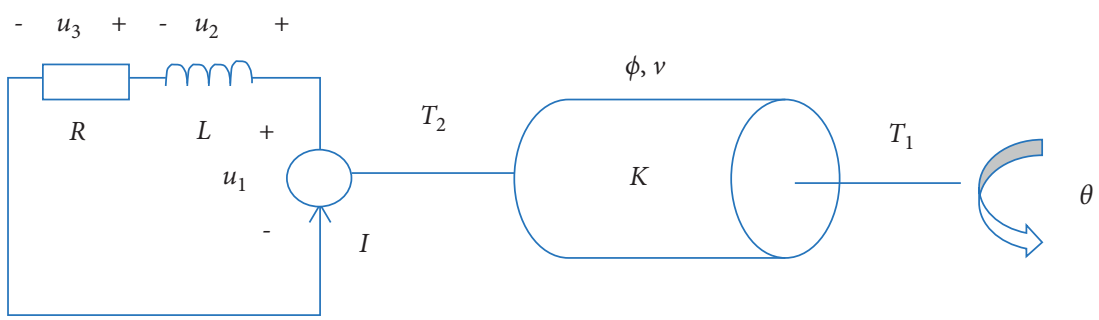

FIGURE 2: A model of generator.

$$
A_{11}=\left[\begin{array}{cc}
1 & 2 \\
-1 & 0
\end{array}\right], A_{12}=\left[\begin{array}{ll}
0 & 0 \\
1 & 1
\end{array}\right], A_{13}=\left[\begin{array}{ll}
1 & 0 \\
0 & 1
\end{array}\right], A_{14}=\left[\begin{array}{ll}
1 & 0 \\
0 & 1
\end{array}\right], A_{21}=\left[\begin{array}{ll}
2 & 1 \\
0 & 1
\end{array}\right],
$$




$$
\begin{aligned}
& A_{22}=\left[\begin{array}{ll}
1 & 0 \\
1 & 0
\end{array}\right], A_{23}=\left[\begin{array}{ll}
1 & 0 \\
0 & 1
\end{array}\right], A_{32}=\left[\begin{array}{ll}
1 & -1 \\
1 & 0
\end{array}\right], A_{33}=\left[\begin{array}{ll}
1 & 0 \\
0 & 1
\end{array}\right], A_{43}=\left[\begin{array}{ll}
1 & 0 \\
0 & 1
\end{array}\right], \\
& B_{1}=\left[\begin{array}{l}
0 \\
1
\end{array}\right], B_{2}=\left[\begin{array}{l}
1 \\
0
\end{array}\right], B_{3}=\left[\begin{array}{c}
0 \\
-1
\end{array}\right], B_{4}=\left[\begin{array}{l}
1 \\
0
\end{array}\right], x_{1}=\left[\begin{array}{l}
T_{1} \\
T_{2}
\end{array}\right], x_{2}=\left[\begin{array}{l}
\mathscr{V} \\
\varnothing
\end{array}\right], x_{3}=\left[\begin{array}{l}
I \\
u_{1}
\end{array}\right], x_{4}=\left[\begin{array}{l}
u_{2} \\
u_{3}
\end{array}\right] .
\end{aligned}
$$

and $\left(A_{43} A_{32} A_{21} A_{14}\right)$ is an invertible matrix.

In the DAE system, variable $x_{4}$ does not appear. Therefore, the algebraic constraint equation (31) can be differentiated with respect to $t$. Then, three times until $x_{4}$ is yielded in the following:

$$
\begin{aligned}
& A_{43} \dot{x}_{3}=B_{4} \dot{\theta}, \\
& A_{43}\left(B_{3} \theta+f_{3}-A_{32} x_{2}-A_{33} x_{3}\right)=B_{4} \dot{\theta}, \\
& -B_{4} \ddot{\theta}+A_{43} B_{3} \dot{\theta}-\left(\mathscr{A}_{4} B_{2}+\mathscr{A}_{5} B_{3}\right) \theta+\mathscr{A}_{4} A_{21} x_{1} \\
& +\left(\mathscr{A}_{4} A_{22}+\mathscr{A}_{5} A_{32}\right) x_{2}+\left(\mathscr{A}_{4} A_{23}+\mathscr{A}_{5} A_{33}\right) x_{3}=0,
\end{aligned}
$$

where $\mathscr{A}_{4}=A_{43} A_{32}, \mathscr{A}_{5}=A_{43} A_{33}$.

Next, one can differentiate equation (27) again for the $t$ and set $c \triangleq\left(A_{43} A_{32} A_{21} A_{14}\right)^{-1}=\left[\begin{array}{cc}0 & 1 \\ 1 / 2 & 0\end{array}\right]$.

Step 1: it is mentioned above that $x_{4}$ is obtained as $x_{4}=L\left(x_{1}, x_{2}, x_{3}\right)+L(\theta, \dot{\theta}, \ddot{\theta})$,

The derivative of the class of the consistent initial conditions is as follows:

$$
\mathfrak{J}^{0}=\left\{\begin{array}{c}
\left(x_{1}\left(t_{0}\right), x_{2}\left(t_{0}\right), x_{3}\left(t_{0}\right)\right) \mid \\
A_{43} x_{3}\left(t_{0}\right)=B_{4} \theta\left(t_{0}\right) \\
\mathscr{A}_{4} A_{21} x_{1}\left(t_{0}\right)+\mathscr{A}_{4} A_{22} x_{2}\left(t_{0}\right)+\mathscr{A}_{4} A_{23} x_{3}\left(t_{0}\right)+\mathscr{A}_{5} A_{32} x_{2}\left(t_{0}\right)+\mathscr{A}_{5} A_{33} x_{3}\left(t_{0}\right) \\
=B_{4} \ddot{\theta}\left(t_{0}\right)-A_{43} B_{3} \dot{\theta}\left(t_{0}\right)+\mathscr{A}_{4} B_{2} \theta\left(t_{0}\right)+\mathscr{A}_{5} B_{3} \theta\left(t_{0}\right)
\end{array}\right\}
$$

Step 2: the following is the definition of the variational function with the class of the consistent initial condition.

$$
\begin{aligned}
J \mathfrak{J}^{0}[x]= & \int_{t_{0}}^{t_{f}} \frac{1}{2}\left[\left[\dot{x}_{1}+A_{1} x_{1}+A_{2} x_{2}+A_{3} x_{3}\right]^{T}\left[\dot{x}_{1}+A_{1} x_{1}+A_{2} x_{2}+A_{3} x_{3}\right]+\right. \\
& \cdot\left[\dot{x}_{2}+A_{21} x_{1}+A_{22} x_{2}+A_{23} x_{3}\right]^{T}\left[\dot{x}_{2}+A_{21} x_{1}+A_{22} x_{2}+A_{23} x_{3}\right]+\left[\dot{x}_{3}+A_{32} x_{2}+A_{33} x_{3}\right]^{T} \\
& \cdot\left[\dot{x}_{3}+A_{32} x_{2}+A_{33} x_{3}\right]+\left[A_{43} x_{3}\right]^{T}\left[A_{43} x_{3}\right]+\left[A_{4} x_{2}+A_{5} x_{3}\right]^{T}\left[A_{4} x_{2}+A_{5} x_{3}\right]+ \\
& \cdot\left[A_{6} x_{1}+A_{2} x_{2}+A_{3} x_{3}\right]^{T}\left[A_{6} x_{1}+A_{2} x_{2}+A_{3} x_{3}\right]+\left[A_{43} x_{3}\left(t_{0}\right)\right]^{T}\left[A_{43} x_{3}\left(t_{0}\right)\right]+ \\
& \cdot\left[A_{4} x_{2}\left(t_{0}\right)+A_{5} x_{3}\left(t_{0}\right)\right]^{T}\left[A_{4} x_{2}\left(t_{0}\right)+A_{5} x_{3}\left(t_{0}\right)\right]+\left[A_{6} x_{1}\left(t_{0}\right)+A_{2} x_{2}\left(t_{0}\right)+A_{3} x_{3}\left(t_{0}\right)\right]^{T} \\
& \left.\cdot\left[A_{6} x_{1}\left(t_{0}\right)+A_{2} x_{2}\left(t_{0}\right)+A_{3} x_{3}\left(t_{0}\right)\right]\right]-\left[\left[F_{1}\right]^{T}\left[\dot{x}_{1}+A_{1} x_{1}+A_{2} x_{2}+A_{3} x_{3}\right]+\right. \\
& \cdot\left[F_{2}\right]^{T}\left[\dot{x}_{2}+A_{21} x_{1}+A_{22} x_{2}+A_{23} x_{3}\right]+\left[F_{3}\right]^{T}\left[\dot{x}_{3}+A_{32} x_{2}+A_{33} x_{3}\right]+\left[F_{4}\right]^{T}\left[A_{43} x_{3}\right]+ \\
& \cdot\left[F_{5}\right]^{T}\left[A_{4} x_{2}+A_{5} x_{3}\right]+\left[F_{6}\right]^{T}\left[A_{6} x_{1}+A_{2} x_{2}+A_{3} x_{3}\right]+\left[F_{4}\left(t_{0}\right)\right]^{T}\left[A_{43} x_{3}\left(t_{0}\right)\right]+\left[F_{5}\left(t_{0}\right)\right]^{T} \\
& \left.\cdot\left[A_{4} x_{2}\left(t_{0}\right)+A_{5} x_{3}\left(t_{0}\right)\right]+\left[F_{6}\left(t_{0}\right)\right]^{T}\left[A_{6} x_{1}\left(t_{0}\right)+A_{2} x_{2}\left(t_{0}\right)+A_{3} x_{3}\left(t_{0}\right)\right]\right] d t
\end{aligned}
$$




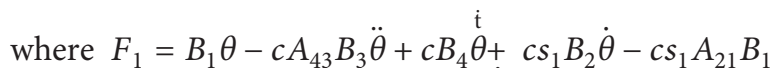
$\theta-c s_{1} A_{22} B_{2} \theta-c s_{1} A_{23} B_{3} \theta+c s_{2} B_{3} \dot{\theta}+c s_{2} A_{32} B_{2} \theta-c s_{2}$ $A_{33} B_{3} \theta, \quad F_{2}=B_{2} \theta, \quad F_{3}=B_{3} \theta, \quad F_{4}=B_{4} \theta, \quad F_{5}=B_{4} \dot{\theta}-$ $A_{43} B_{3} \theta, F_{6}=B_{4} \theta-A_{43} B_{3} \dot{\theta}+s_{1} B_{2} \theta+s_{2} B_{3} \theta$.
Step 3: one can set $x_{11}(t)=\sum_{i=0}^{5} a_{i} \varphi_{i}(t), x_{12}(t)=\sum_{i=0}^{5}$ $b_{i} \varphi_{i}(t)$,

$x_{21}(t)=\sum_{i=0}^{5} c_{i} \varphi_{i}(t), x_{22}(t)=\sum_{i=0}^{5} d_{i} \varphi_{i}(t), x_{31}(t)=\sum_{i=0}^{5}$ $v_{i} \varphi_{i}(t)$, and $x_{32}(t)=\sum_{i=0}^{5} w_{i} \varphi_{i}(t)$, with $\varphi_{i}(t)=t^{i}$ to obtain

$$
x_{4}(t)=L\left(\sum_{i=0}^{5} a_{i} t^{i}, \sum_{i=0}^{5} b_{i} t^{i}, \sum_{i=0}^{5} c_{i} t^{i}, \sum_{i=0}^{5} d_{i} t^{i}, \sum_{i=0}^{5} v_{i} t^{i}, \sum_{i=0}^{5} w_{i} t^{i}, t\right)+L(\theta, \dot{\theta}, \ddot{\theta}, \theta \bigotimes) .
$$

Step 4: $J$ is a function of $\vec{a}, \vec{b}, \vec{c}, \vec{d}, \vec{v}$, and $\vec{w}$, and as $J(\vec{a}, \vec{b}, \vec{c}, \vec{d}, \vec{v}, \vec{w})$ is extremized, one can set $\partial J /$ $\partial \vec{a}=0, \partial J / \partial \vec{b}=0, \partial J / \partial \vec{c}=0, \partial J / \partial \vec{d}=0, \partial J / \partial \vec{v}=0$, and $\partial J / \partial \vec{w}=0$. It is led to the linear algebraic equation $\mathrm{M}(\vec{a} \vec{b} \vec{c} \vec{d} \vec{v} \vec{w})=\mathrm{N}$, which is solvable directly for $(\vec{a} \vec{b} \vec{c} \vec{d} \vec{v} \vec{w})=\mathrm{M}^{-1} \mathrm{~N}$. Hence, the approximate solution $\left(x_{1}(t), x_{2}(t), x_{3}(t), x_{4}(t)\right)$ is determined. Lastly, the derivative of $J$ with respect to $\vec{a}, \vec{b}, \vec{c}$, $\vec{d}, \vec{v}$, and $\vec{w}$ is used to obtain the matrix coefficient $M$.

Step 5: to evaluate the solution's accuracy without knowing the exact solution, the model utilises $L_{2}$-norm by substituting the values of $x_{11}(t)=\sum_{i=0}^{5} a_{i} t^{i}$, $x_{12}(t)=\sum_{i=0}^{5} b_{i} t^{i}, \quad x_{21}(t)=\sum_{i=0}^{5} c_{i} t^{i}, x_{22}(t)=\sum_{i=0}^{5} d_{i} t^{i}$, $x_{31}(t)=\sum_{i=0}^{5} v_{i} t^{i}$, and $x_{32}(t)=\sum_{i=0}^{5} w_{i} t^{i}$ in system (31). The error presented in the test example at each point along time $t \in[0,1]$ and for different kinds of $\theta$ are displayed (Figures 3-6).

4.3. Application of the Rotating Masses System. Consider a system of two rotating masses that are described by the torques $T_{1}(t), T_{2}(t), T_{3}(t)$, and $T_{4}(t)$ and the angular velocities $\alpha_{1}(t)$ and $\alpha_{2}(t)$. The masses have the moments of inertia $\gamma_{1}$ and $\gamma_{1}$. Figure 7 illustrates this scenario.

The system to matrically describes this model is provided as

$$
\left[\begin{array}{cccc}
\gamma_{1} & 0 & 0 & 0 \\
0 & \gamma_{2} & 0 & 0 \\
0 & 0 & 1 & 0 \\
0 & 0 & 0 & 0
\end{array}\right]\left[\begin{array}{c}
\dot{\alpha}_{1} \\
\dot{\alpha}_{2} \\
\dot{T}_{2} \\
\dot{T}_{3}
\end{array}\right]+\left[\begin{array}{cccc}
0 & 0 & 1 & 1 \\
1 & 0 & 1 & 0 \\
0 & -1 & -1 & 0 \\
0 & 0 & 1 & 0
\end{array}\right]\left[\begin{array}{c}
\alpha_{1} \\
\alpha_{1} \\
T_{2} \\
T_{3}
\end{array}\right]=\left[\begin{array}{ll}
1 & 0 \\
0 & 0 \\
0 & 0 \\
0 & 1
\end{array}\right]\left[\begin{array}{l}
T_{1} \\
T_{4}
\end{array}\right],
$$

with $\gamma_{1}=\gamma_{2}=1$ and $T_{1}=t^{5}-4 t, T_{4}=2 t^{2}+3$.

Step 1: since $A_{43} A_{32} A_{21} A_{14}$ exists as an invertible matrix, the variable $T_{3}$ does not appear and $\dot{T}_{2}=4 t$ through differentiation of the algebraic constraint of

system (39), we can continue to differentiate until $T_{3}$ obtained as $T_{3}=\alpha_{1}+t^{5}-4 t$ with consistent initial condition:

$$
\mathfrak{J}^{0}=\left\{\left(\alpha_{1}\left(t_{0}\right), \alpha_{2}\left(t_{0}\right), T_{2}\left(t_{0}\right)\right) \mid T_{2}=2 t_{0}^{2}+3, \alpha_{2}+T_{2}=4 t_{0},-\alpha_{1}+\alpha_{2}=4\right\} .
$$

Step 2: using the variational formulation associated with this consistent initial condition,

$$
\begin{aligned}
J_{\widetilde{J}^{0}}[x]= & \int_{t_{0}}^{t_{f}} \frac{1}{2}\left[\left[\dot{\alpha}_{1}+T_{2}+T_{3}\right]^{T}\left[\dot{\alpha}_{1}+T_{2}+T_{3}\right]+\left[\dot{\alpha}_{2}+\alpha_{1}+T_{2}\right]^{T}\left[\dot{\alpha}_{2}+\alpha_{1}+T_{2}\right]\right. \\
& \left.\cdot+\left[\dot{T}_{2}-\alpha_{2}-T_{2}\right]^{T}\left[\dot{T}_{2}-\alpha_{2}-T_{2}\right]+\left[T_{2}\right]^{T}\left[T_{2}\right]+\left[\alpha_{2}+T_{2}\right]^{T}\left[\alpha_{2}+T_{2}\right]+\left[-\alpha_{1}+\alpha_{2}\right]^{T}\left[-\alpha_{1}+\alpha_{2}\right]\right]- \\
& \cdot\left[\left[t^{5}-4 t\right]^{T}\left[\dot{\alpha}_{1}+T_{2}+T_{3}\right]+\left[2 t^{2}+3\right]^{T}\left[T_{2}\right]+4 t\left[\alpha_{2}+T_{2}\right]+4\left[-\alpha_{1}+\alpha_{2}\right]\right] d t .
\end{aligned}
$$




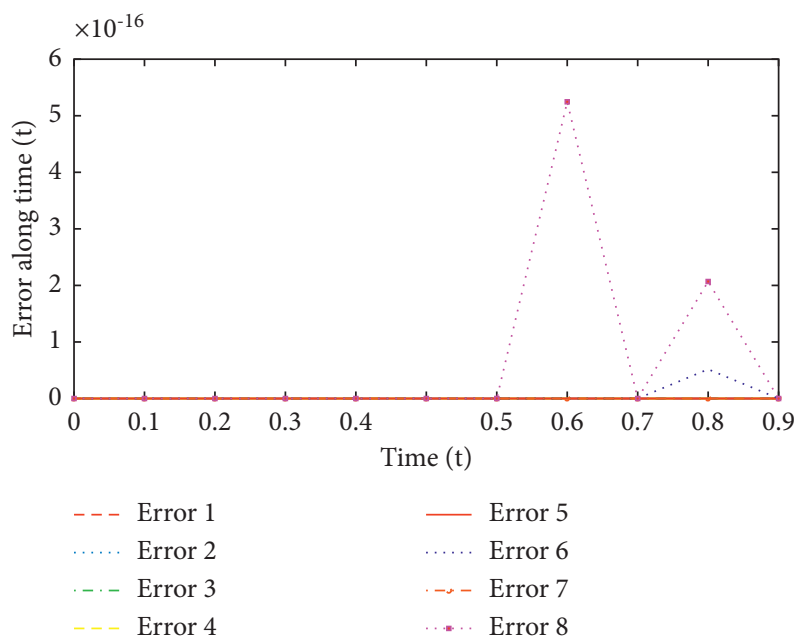

Figure 3: The error of the generator system solution for $\theta=e^{-t}$.

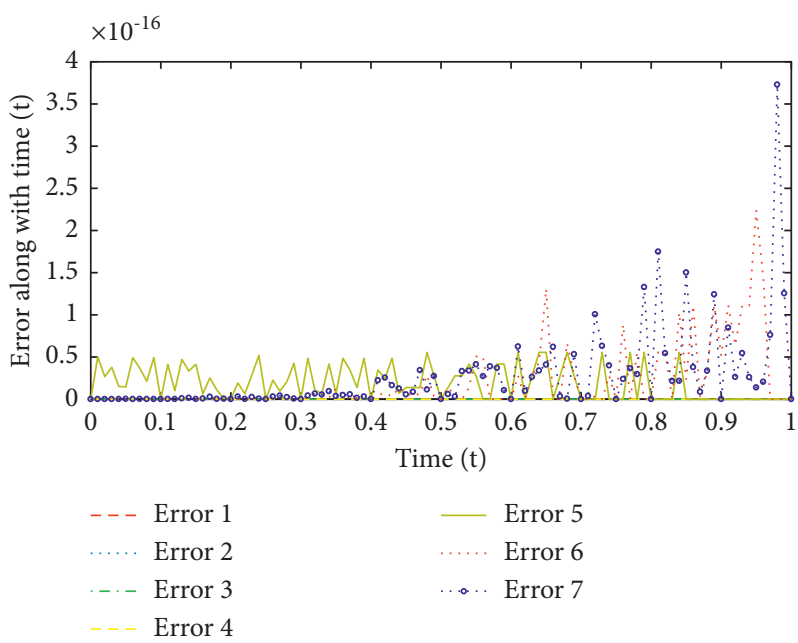

Figure 4: The error of the generator system solution for $\theta=\sin t$.

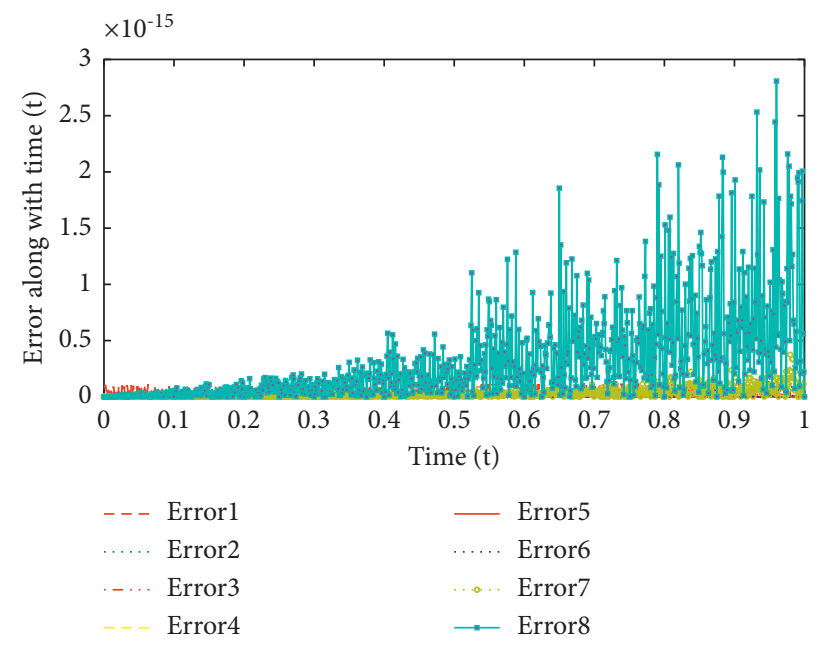

FIgURE 5: The error of the generator system solution for $\theta=\sin t+\cos t$. 


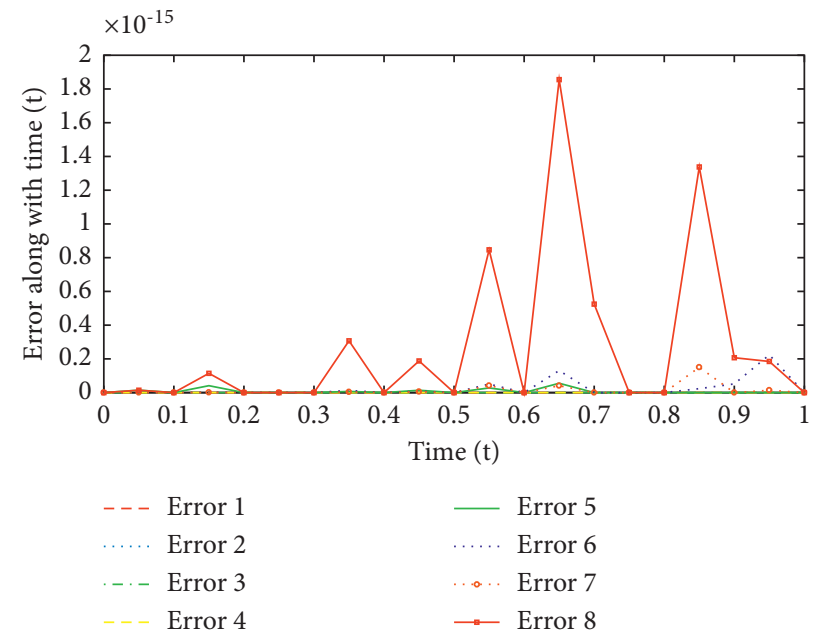

Figure 6: The error of the generator system solution for $\theta=t^{4}+t^{2}$.

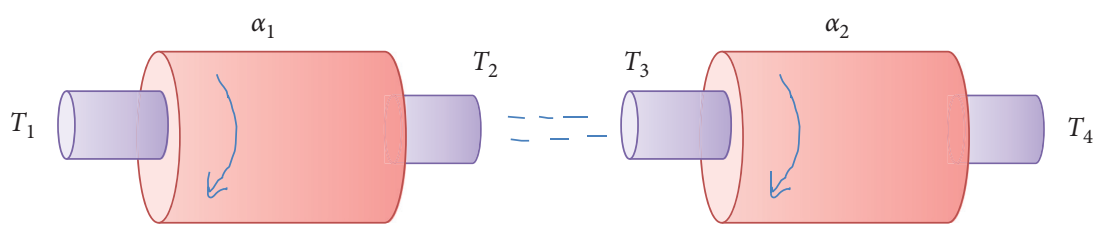

FIGURE 7: Connected rotating masses.

One can set $\alpha_{1}(t)=\sum_{i=0}^{5} p_{i} t^{i}, \alpha_{2}(t)=\sum_{i=0}^{5} \eta_{i} t^{i}, T_{2}(t)=$ $\sum_{i=0}^{5} \mu_{i} t^{i}$ to obtain

$$
T_{3}=L\left(\sum_{i=0}^{5} p_{i} t^{i}, \sum_{i=0}^{5} \eta_{i} t^{i}, \sum_{i=0}^{5} \mu_{i} t^{i}, t\right)+L\left(T_{1}, T_{4}, \dot{T}_{4}, \ddot{T}_{4}, \stackrel{\dot{t}}{T_{4}}\right) .
$$

Step 3: as mentioned above, the functional $J$ is a function of $\vec{p}, \vec{\eta}$, and $\vec{\mu}$. As $J(\vec{p}, \vec{\eta}, \vec{\mu})$ is extremized, one can set $\partial J / \partial \vec{p}=0, \partial J / \partial \vec{\eta}=0$, and $\partial J / \partial \vec{\mu}=0$. This yields the linear algebraic equation
$\mathrm{M}(\vec{p} \vec{\eta} \vec{\mu})=\mathrm{N}$, which is solvable directly for $(\vec{p} \vec{\eta} \vec{\mu})=\mathrm{M}^{-1} \mathrm{~N}$. Hence, the approximate solution $\left(\alpha_{1}(t), \alpha_{2}(t), T_{2}(t)\right)$ is determined. The derivative of $J$ with respect to $\vec{p}, \vec{\eta}$, and $\vec{\mu}$ is used to obtain the matrix coefficient $M$.

Step 4: to evaluate the solution's accuracy without knowing the exact solution, we use $L_{2}$-norm where the values of $\alpha_{1}(t)=\sum_{i=0}^{5} p_{i} t^{i}, \alpha_{2}(t)=\sum_{i=0}^{5} \eta_{i} t^{i}$, and $T_{2}(t)=\sum_{i=0}^{5} \mu_{i} t^{i}$ are substituted in system (39).

For differential equations,

$$
\begin{aligned}
\left\|\dot{\alpha}_{1}+T_{2}+T_{3}-t^{5}+4 t\right\|_{2} & =\left[\int_{0}^{1}\left|\dot{\alpha}_{1}+T_{2}+T_{3}-t^{5}+4 t\right|^{2} d t\right]^{1 / 2}=0.0035 \\
\left\|\dot{\alpha}_{2}+\alpha_{1}+T_{2}\right\|_{2} & =\left[\int_{0}^{1}\left|\dot{\alpha}_{2}+\alpha_{1}+T_{2}\right|^{2} d t\right]^{1 / 2}=2 \times 10^{-5} \\
\left\|\dot{T}_{2}-\alpha_{2}-T_{2}\right\|_{2} & =\left[\int_{0}^{1}\left|\dot{T}_{2}-\alpha_{2}-T_{2}\right|^{2} d t\right]^{1 / 2}=7 \times 10^{-3}
\end{aligned}
$$


For equality algebraic constraint, $\left[\int_{0}^{1}\left|T_{2}-2 t^{2}-3\right|^{2} d t\right]^{1 / 2}$ $=2 \times 10^{-3}$.

\section{Results and Discussion}

Using the test example to discuss and validate the presented method for the system of index-four Hessenberg differential algebraic equations, the results indicate how the exact and the approximate solutions match exceedingly well in a way that demonstrates the accuracy and efficiency of the model. Applications 4.1 and 4.2 show to employ differential algebraic equations as a modelling environment in system applications.

In examples where a state space cannot effectively describe the whole system, the model system will be reduced to a standard state-space system on the manifold of the resulting algebraic constraints which is provide a simplest system to deal with and this reduction reduce accuracy of the results. These examples are taken as a test for the proposed method. A step-by-step implementation, the approximate solution is parameterised via polynomial base functions, which are dense in $C\left[I, R^{3}\right]$. Despite reasonably small numbers of these polynomials with an unknown coefficient, the obtained solutions prove to be rather accurate and efficient.

\section{Conclusions}

This research presented an efficient and reliable to solve higher index (index four) linear Hessenberg control DAEs. To ensure a controllable and linearly independent set of functions in its base, the setting space of the solvability is assumed to be the separable Banach space. The differentiation index is adopted and used repeatedly in this study until we achieve the required conditions around the implicit function theorem. This outcome depends on the index of the system. Reduced Hessenberg DAEs are obtained with the help of the implicit function theorem. An operator form has been used to write the latest system. For this case, we developed the variational formulation. The representation of the solution has been as a linear combination of elements in the setting space. The cost for utilising these new measures consists of more smoothness conditions on the control signal.

Generally, the treating of the system has been as a perturbation in the nonhomogenous functions. The example, index four, is efficiently illustrated. Despite a rather simple basis function type, the evaluation of the approach revealed excellent accuracy Due to the decision-maker, these bases can be modified to include spline and orthonormal basis.

\section{Data Availability}

The data used in the article are generated by Octave and can be made available upon request.

\section{Conflicts of Interest}

The author declares that there are no conflicts of interest regarding the publication of this paper.

\section{Acknowledgments}

The authors would like to thank Mustansiriyah University (http://www.uomustansiriyah.edu.iq) Baghdad, Iraq, for its support of this work.

\section{References}

[1] F. Awawdeh, H. M. Jaradat, and O. Alsayyed, "Solving system of daes by homotopy analysis method," Chaos, Solitons \& Fractals, vol. 42, no. 3, pp. 1422-1427, 2009.

[2] B. I. Akinnukawe, O. A. Akinfenwa, and S. A. Okunuga, "Hybrid block algorithm for solving differential algebraic equations with hessenberg index 3," FUTA Journal of Research in Sciences, vol. 15, no. 1, pp. 32-39, 2019.

[3] E. Çelık, "On the numerical solution of differential-algebraic equations with index-2," Applied Mathematics and Computation, vol. 156, no. 2, pp. 541-550, 2004.

[4] P. Kunkel and V. Mehrmann, "A new class of discretization methods for the solution of linear differential-algebraic equations with variable coefficients," SIAM Journal on $\mathrm{Nu}$ merical Analysis, vol. 33, no. 5, pp. 1941-1961, 1996.

[5] W. S. Martinson and P. I. Barton, "A differentiation index for partial differential-algebraic equations," SIAM Journal on Scientific Computing, vol. 21, no. 6, pp. 2295-2315, 2000.

[6] U. M. Ascher and P. Lin, "Sequential regularization methods for higher index daes with constraint singularities: the linear index-2 case," SIAM Journal on Numerical Analysis, vol. 33, no. 5, pp. 1921-1940, 1996.

[7] M. Golchian, M. gachpazan, and S. H. tabasi, "A new approach for computing the exact solutions of daes in generalized hessenberg forms," International Journal of Nonlinear Analysis and Applications, vol. 11, no. 1, pp. 199-206, 2020.

[8] M. Karta and E. Celik, "On the numerical solution of differential algebraic equations with hessenberg index-3," Discrete Dynamics in Nature and Society, vol. 2012, Article ID 147240, 12 pages, 2012.

[9] P. Lin and R. J. Spiteri, "A predicted sequential regularization method for index-2 hessenberg daes," SIAM Journal on Numerical Analysis, vol. 39, no. 6, pp. 1889-1913, 2002.

[10] R. A. Zaboon and G. F. Abd, "Approximate solution of a linear descriptor dynamic control system via a non-classical variational approach," IOSR -Journal of Mathematics, vol. 11, no. 1, pp. 1-11, 2015.

[11] G. F. Abd and R. A. Zaboon, "Solution of time-varying index2 linear differential algebraic control systems via A variational formulation technique," Iraqi Journal of Science, vol. 62, no. 10, pp. 3656-3671, 2021.

[12] G. F. Abd and R. A. Zaboon, "Parameterization technique for solving variational formulation of index three linear timevarying singular control systems," Journal of Interdisciplinary Mathematics, vol. 24, no. 7, pp. 1935-1945, 2021.

[13] S. Shimizu and N. Matubayasi, "Implicit function theorem and Jacobians in solvation and adsorption," Physica A: Statistical Mechanics and Its Applications, vol. 570, Article ID 125801, 2021.

[14] D. Idczak, "A global implicit function theorem and its applications to functional equations," Discrete and Continuous Dynamical Systems B, vol. 19, no. 8, pp. 2549-2556, 2014.

[15] G. R. Duan, Analysis and Design of Descriptor Linear Systems, Springer Science and Business Media, Berlin, Germany, 2010. 